\title{
Comparison of five latex agglutination kits for detecting group A streptococcal antigen
}

\author{
J.R. BURDICK, PhD \\ WILLIAM SOTTILE, PhD \\ Chicago, Illinois
}

\begin{abstract}
The sensitivity and specificity of five latex agglutination kits for rapid detection of group A Streptococcus (GAS) antigen in direct throat swabs were compared. A total of 1,007 clinical specimens for diagnosis of GAS pharyngitis were randomly tested against one of the kits as well as by standard cultural methods. B-hemolytic streptococci were isolated from 291 specimens $(29 \%)$. Of these isolates $128(44 \%)$ were presumptively identified as GAS by bacitracin susceptibility and $129(44 \%)$ were identified as GAS by serotyping. The sensitivity value of the rapid detection kits in comparison with standard cultural methods ranged from $60 \%$ to $68 \%$; the specificity ranged from $91 \%$ to $98 \%$; the positive predictive value ranged from $43 \%$ to $80 \%$, and the negative predictive value ranged from $94 \%$ to $96 \%$. When tested in vitro with varying dilutions of antigen, the test kits did not differ substantially in detecting the low concentrations of antigen.
\end{abstract}

Classically, the definitive diagnosis of streptococcal pharyngitis has been made by isolation and identification of group A B-hemolytic streptococci from pharyngeal specimens. Presumptive identification of this organism is made by demonstrating in vitro susceptibility to 0.04 units of bacitracin. ${ }^{1}$ Unfortunately, this method of presumptive identification often yields incorrect results: false-positive because occasional group $\mathrm{C}$ streptococci are sensitive to bacitracin, and false-negative because of fail- ure to detect $\beta$-hemolysis in oxygen-sensitive strains of group A streptococci. ${ }^{2}$ Some authors ${ }^{3}$ have reported discrepant results in which one inoculated agar plate yielded moderate to heavy growth of B-hemolytic streptococci and a second plate, inoculated from the same throat swab, showed no such growth. ${ }^{3}$ The standard Lancefield precipitation test for serologic identification of the B-hemolytic streptococci ${ }^{4}$ remains the reference standard for identification; however, the procedure is time consuming and expensive. Newer serologic procedures employing a latex agglutination immunoassay are less time consuming and are as sensitive and specific as the Lancefield precipitation technique; however, they are more expensive than the bacitracin method commonly employed by most laboratories. ${ }^{5}$

Interest in immunologic procedures that directly detect infectious agents in specimens without cultivation has been increasing. ${ }^{6-9}$ Several kits for direct detection of group A streptococcal antigen in oropharyngeal swabs are now marketed. Most of the kits are based on a latex particle agglutination immunoassay and differ in their antigen extraction method, that is, acid ${ }^{10-11}$ or enzymatic. ${ }^{12-13}$ They also differ in minimum time for test results, ranging from 5 to 60 minutes. Most of these kits have been evaluated singly by comparison to standard cultural techniques, ${ }^{10-18}$ and a few two-kit comparative trials have been reported. ${ }^{15,19}$ The usefulness and importance of comparative evaluations of both antigen and antibody detection kits is amply evidenced in the literature. ${ }^{20-24}$ Therefore the purpose of this study was to conduct a direct comparison of the sensitivity, specificity, and the predictive values (positive and negative) of five commercially available group A streptococci direct de- 
tection kits, and compare the efficiency of each kit to the standard culture techniques of a single testing laboratory.

\section{Methods}

Throat specimens were obtained from outpatients with suspected GAS pharyngitis by swabbing the tonsillar region and posterior pharynx with a rayontipped swab (Culturette, Marion Scientific, Kansas City, Mo). All specimens were submitted to the microbiology laboratory to be routinely screened for streptococcal pharyngitis.

The swab for each patient was inoculated on trypticase soy agar supplemented with $5 \%$ sheep blood (BBL Microbiology Systems, Cockeysville, Md). The specimen was streaked for isolation, and the agar was stabbed across the primary streak. Cultures were incubated aerobically at $35^{\circ} \mathrm{C}$ in $5 \% \mathrm{CO}_{2}$ for 18 to 24 hours. $\beta$-hemolytic streptococcal colonies were presumptively identified as small ( 0.5 to 1.0 $\mathrm{mm}$ ), white-gray colonies surrounded by a clear zone of hemolysis. $\beta$-hemolytic colony-forming units (CFU) of streptococci were quantified as follows: rare, less than 10 colonies per plate; few, 10 to 50 colonies per plate; moderate, 50 to 100 colonies per plate; and many, more than 100 colonies per plate. B-hemolytic colonies were subcultured from the primary plate, tested for bacitracin susceptibility according to standard methods, ${ }^{1}$ and identified definitively by serologic techniques (Streptex; Wellcome Diagnostics, Research Triangle Park, NC).

The same swab submitted for culture was also used for detection of GAS antigen after inoculation of agar plate. At the beginning of each collection day, a direct latex agglutination kit was chosen by random number for that day's testing. The kits tested are shown in Table 1. Strict adherence to the protocol specified by each manufacturer was followed. All the kits used in this study were generously supplied by the manufacturers.

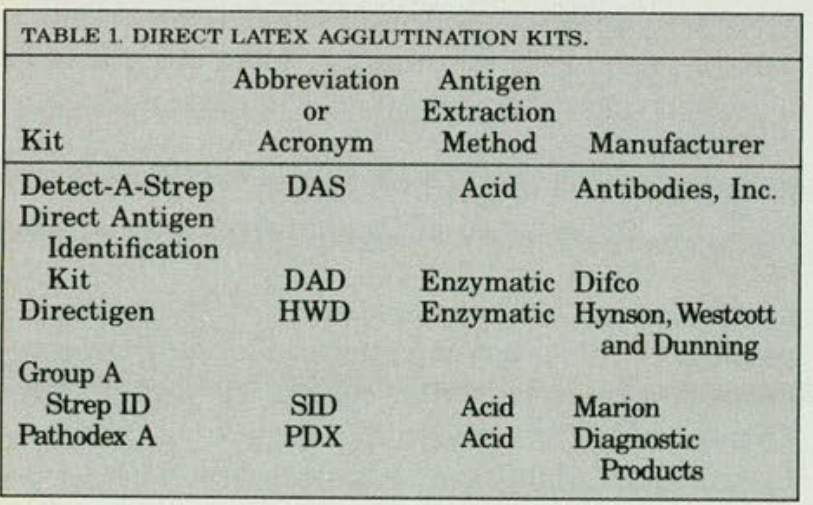

The relative sensitivity of each kit was determined according to a modification of the procedure described by Petts. ${ }^{8}$ Briefly, an overnight broth culture of a clinical isolate of GAS was diluted tenfold in sterile saline solution, and colony counts were performed in triplicate for each dilution. Sterile swabs were placed in each dilution and allowed to absorb fluid, removed, drained, and subsequently treated as a clinical throat swab for GAS antigen detection. The volume of fluid absorbed by each swab was determined by weighing before and after loading.

Analysis of the data to determine sensitivity, specificity, predictive value for a positive result and predictive value for a negative result was done by the method of Griner et al. ${ }^{25}$ The $x^{2}$ test was used to compare discrete variables.

\section{Results}

Oropharyngeal specimens were collected from 1,007 patients with pharyngitis and were processed according to the procedure described. $ß$-hemolytic streptococci were recovered from 291 (29\%) specimens by cultural methods. The predominant serogroups identified were group A, accounting for $44 \%$ (129/291), and group C, accounting for 32\% (93/ 291 ) of the $\beta$-hemolytic streptococcal isolates. The percentage of isolates in each of groups B, F, and $\mathrm{G}$ were $8 \%, 9 \%$, and $6 \%$, respectively. Two $1 \%$ isolates were group D and were identified serologically only after the antigen-enzyme extraction mixture was incubated an additional $30 \mathrm{~min}$. Of the 129 serologically identified group A isolates, 115 were presumptively identified to be GAS by bacitracin susceptibility (sensitivity, 89\%), and 149 of the 162 non-group $\mathrm{A}$ isolates were resistant to bacitracin (specificity, 92\%). Overall, bacitracin tests were in $91 \%(264 / 291)$ agreement with serologic identification of GAS.

A comparison of each kit with standard culture methods for detecting GAS on the basis of serologic identification is shown in Table 2. The total number of tests for each kit represents, to the extent possible, random selection of a kit for a given day's testing and ranged from 169 to 240 tests. A total of 49 test kit results were false positive with $71 \%$ (35/49) occurring in specimens that produced no $\beta$-hemolytic CFU on culture. The serotype distribution of the non-group A isolates representing the remaining 14 false positives did not appear to be substantially different from the general distribution observed for all cultures yielding B-hemolytic CFU. The comparative sensitivities, specificities, positive predictive values, and negative predictive values are given in Fig 1 . The greatest variation in the performance of the test kits occurred 


\begin{tabular}{|c|c|c|c|c|}
\hline $\begin{array}{l}\text { Test } \\
\text { Kit }\end{array}$ & $\begin{array}{l}\text { Kit } \\
\text { Results } \\
\end{array}$ & No. Positive & $\begin{array}{r}\text { Culture Result } \\
\text { No. Negative }\end{array}$ & Total \\
\hline DAS & $\begin{array}{l}\text { Positive } \\
\text { Negative } \\
\text { Total }\end{array}$ & $\begin{array}{r}13 \\
7 \\
20\end{array}$ & $\begin{array}{r}14 \\
150 \\
164\end{array}$ & $\begin{array}{r}27 \\
157 \\
184\end{array}$ \\
\hline $\mathrm{DAD}$ & $\begin{array}{l}\text { Positive } \\
\text { Negative } \\
\text { Total }\end{array}$ & $\begin{array}{l}21 \\
\frac{10}{31}\end{array}$ & $\begin{array}{r}6 \\
183 \\
189\end{array}$ & $\begin{array}{r}27 \\
193 \\
220\end{array}$ \\
\hline HWD & $\begin{array}{l}\text { Positive } \\
\text { Negative } \\
\text { Total }\end{array}$ & $\begin{array}{r}12 \\
\frac{8}{20}\end{array}$ & $\begin{array}{r}3 \\
146 \\
149\end{array}$ & $\begin{array}{r}15 \\
154 \\
169\end{array}$ \\
\hline SID & $\begin{array}{l}\text { Positive } \\
\text { Negative } \\
\text { Total }\end{array}$ & $\begin{array}{l}20 \\
\frac{12}{32}\end{array}$ & $\begin{array}{r}10 \\
198 \\
208\end{array}$ & $\begin{array}{r}30 \\
210 \\
240\end{array}$ \\
\hline PDX & $\begin{array}{l}\text { Positive } \\
\text { Negative } \\
\text { Total }\end{array}$ & $\begin{array}{l}\frac{12}{8} \\
20\end{array}$ & $\begin{array}{r}16 \\
158 \\
174\end{array}$ & $\begin{array}{r}28 \\
166 \\
194\end{array}$ \\
\hline
\end{tabular}

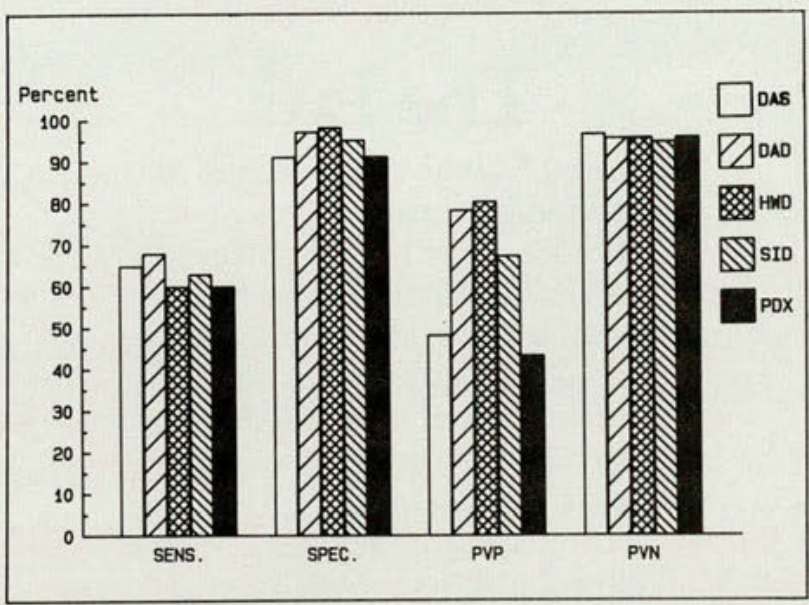

Fig. 1. Comparison of the sensitivity (SENS), specificity (SPEC), and predictive values for positive results $(P V P)$ and for negative results (PVN) of the five test kits for detection of GAS in throat swabs.

in the positive predictive values, which ranged from $43 \%$ (PDX) to $80 \%$ (HWD). No significant differences among the kits with regard to sensitivity (60\% to $68 \%)$, specificity, (91\% to $98 \%$ ) or negative predictive value (94\% to $96 \%$ ) were shown.

Table 3 summarizes the individual performances of each kit for the in vitro testing of the lower limits of antigen detection. Tenfold dilutions were made from a broth culture of GAS containing 3.0 $\times 10^{6} \mathrm{CFU} / \mathrm{mL}$. Swabs were placed in the dilutions and the volume retained by the swab was calculated from the increased weight and ranged from 0.159 to $0.178 \mathrm{~mL}$ with a mean of $0.168 \pm 0.01$ $\mathrm{mL}$. The results showed that each test kit gave a positive reaction at a dilution of $10^{-3}(460 \mathrm{CFU} /$ swab). The PDX kit showed a borderline positive result at the $10^{-4}$ dilution.

\section{Discussion}

The predominant B-hemolytic Streptococcus isolated in this study was group A, which accounted for $44 \%$ of the isolates. The most common non-A B-hemolytic Streptococcus was group C, which accounted for $32 \%$ of all $\beta$-hemolytic streptococci and $57 \%$ of all non-A $B$-hemolytic streptococci. The distribution of non-group $\mathrm{A}$ isolates from patients with pharyngitis varies in the literature, although group $\mathrm{C}^{26}$ and group $\mathrm{G}^{27}$ frequently predominate as the most common non-group $\mathrm{A}$ isolates. Both group $\mathrm{C}^{28}$ and group $\mathrm{G}^{29}$ streptococci can cause a pharyngitis that is clinically indistinguishable from illness associated with the group A streptococci. Some authors argue that patients with group C streptococcal infection should undergo treatment so that local and metastatic suppurative complications can be avoided ${ }^{30}$; however, other evidence has shown that therapy neither significantly modifies the severity nor affects the duration of symptoms associated with non-group A streptococcal pharyngitis. ${ }^{31}$ Perhaps the evidence should be re-examined in light of recent findings that early administration of antimicrobics for group A streptococcal pharyngitis alters the course of disease. ${ }^{32}$

The standard cultural methods used in this study included serologic identification of $\beta$-hemolytic streptococcal isolates as the reference method. The sensitivity of presumptive identification by bacitracin susceptibility as compared to serologic identification was $89 \%$. Among the 13 false-positive bacitracin-sensitive strains, group $\mathrm{C}$ accounted for 10 $(77 \%)$ of the isolates, and group $\mathrm{G}$ accounted for the remaining 3 isolates ( $23 \%)$. The serotype distribution of the false-positive bacitracin-suscepti- 


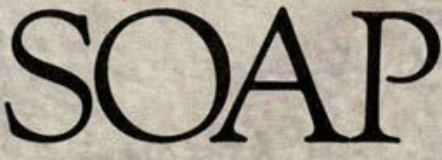

\section{The Truth:}

DOVE is not a soap...but a neutral cleansing bar with $1 / 4$ moisturizing cream. With a neutral $\mathrm{pH}$ of 7, DOVE does not alter the skin's natural buffering capacity. Rather than strip away the skin's moistureprotecting oils the way soaps do, DOVE works to retain needed moisture. DOVE is milder than soap; clinical tests prove it. No wonder physicians recommend DOVE more than any other cleansing product.

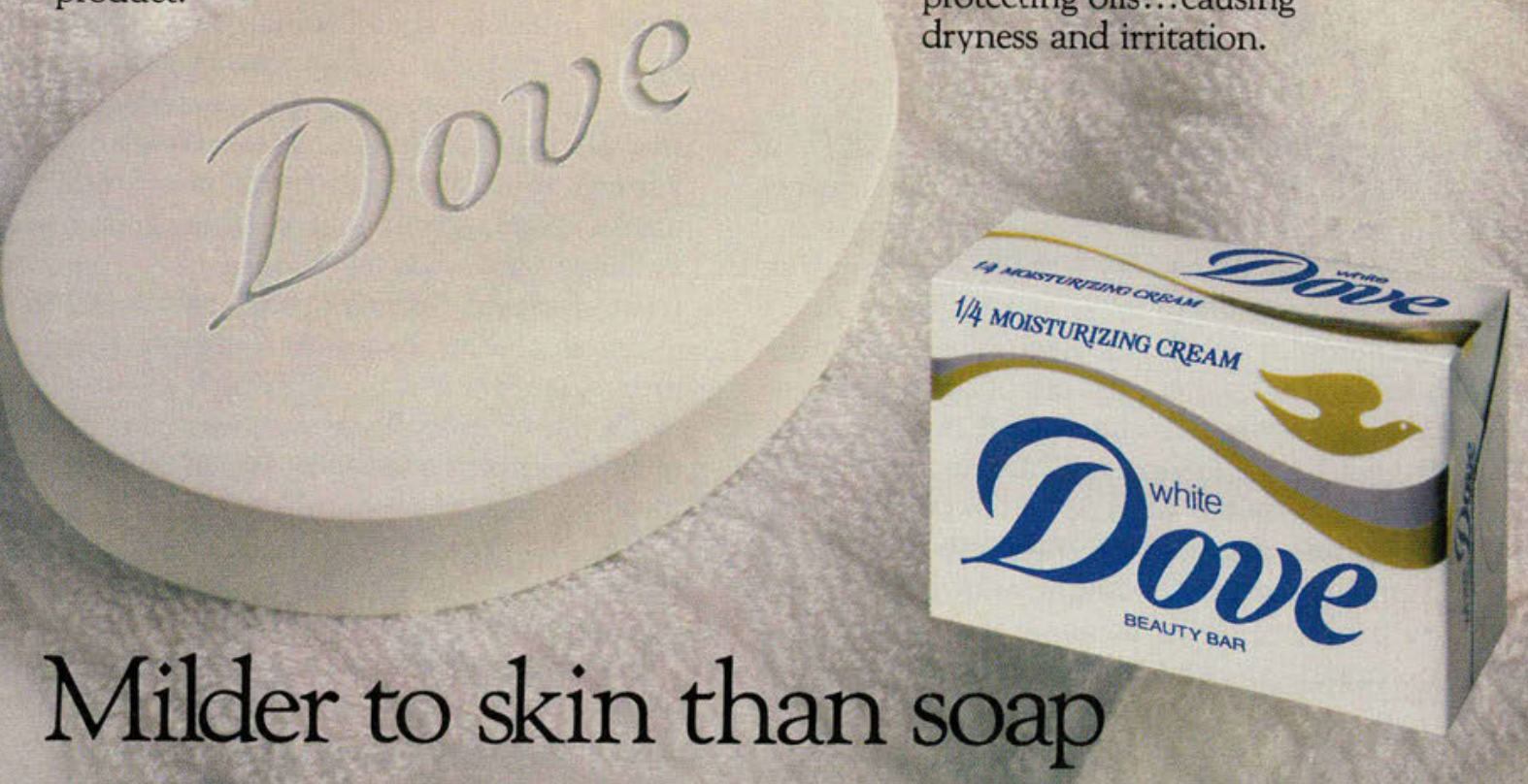

\section{The Lye:}

Though some soaps profess mildness, all soaps are made with lye. That's what makes soap alkaline by definition. Despite the addition of various emollients, soaps remain decidedly alkaline. Even translucent soap bars, though milder looking, are in the region of $\mathrm{pH} 10$. This helps explain why soap can alter the skin's protective acid mantle. It also explains why frequent soaping can actually strip away the skin's moistureprotecting oils...causing dryness and irritation. 
ble isolates was significantly different from that observed for all isolates $(\mathrm{P}<.001)$. Therefore, the use of bacitracin sensitivity to evaluate direct latex agglutination kits for GAS would have limitations in studies yielding a high proportion of group $\mathrm{C}$ and group $\mathrm{G}$ isolates. Such isolates could increase the number of false negative results (bacitracin sensitive, latex negative), thereby decreasing the sensitivity value.

The sensitivity of the test kits varied from 60 to $68 \%$. Generally, our findings were in the lower range of reported sensitivities for comparative studies on direct latex agglutination kits. ${ }^{10-18}$ However, previous studies have shown that sensitivity of a single kit may range from $62 \%{ }^{10}$ to $95 \% .{ }^{18}$ This variation reflects, in part, variation among laboratories in specimen collection and standard culturing techniques. Throat culture technology is not sufficiently standardized; therefore, comparisons among studies are difficult. ${ }^{33}$ In our study all kits were evaluated by use of the uniform standard techniques of a single laboratory, so that the cultural methods used to evaluate kit performance had some standardization or uniformity. Under these conditions, no significant differences in either sensitivity or specificity were found among the five DLAKs tested.

A possible explanation for decreased sensitivity would include the use, in our study, of a singleswab specimen for both culture and the antigen detection tests. The literature includes conflicting reports that culture plate inoculation may sufficiently deplete the swab of GAS as to preclude a positive antigen detection test. $3,10,33$ Poorly collected specimens further increase the likelihood of false negative DLAK results. ${ }^{10}$ One study has shown that a minimum of $2 \times 10^{5} \mathrm{CFU}$ of GAS per swab is necessary for a positive latex test. ${ }^{34}$ These data differ significantly from our findings of a positive latex agglutination reaction for swabs containing $4.6 \times 10^{2} \mathrm{CFU}$ of GAS. Nevertheless, $67 \%(30 / 45)$ of false-negative latex agglutination test results occurred from specimens that yielded less than 50 ß-hemolytic CFU on primary culture.

The positive predictive values of the kits differed significantly. Kits (DAD and HWD) that utilize an enzymatic extraction method for release of GAS antigen had substantially higher predictive values of positivity than those using an acid extraction method. The positive predictive values showed that between $20 \%$ to $57 \%$ of the patients diagnosed as having GAS pharyngitis by direct antigen detection tests would receive unnecessary antimicrobic therapy. The decision whether to use a direct antigen detection test as a primary diagnostic tool as the basis for therapy should depend on whether a
TABLE 3. IN VITRO COMPARISON OF LOWER LIMIT OF GAS ANTIGEN DETECTION BY TEST KITS. DILUTIONS ARE FROM CULTURE CONTAINING $3.0 \times 10^{6} \mathrm{CFU} / \mathrm{ML}$ STREPTOCOCCUS PYOGENES.

\begin{tabular}{lllllll} 
& & & \multicolumn{3}{c}{ Test Kit Result* } \\
Dilutions & CFU/swab & DAS & DAD HWD PDX & SID \\
$10^{-2}$ & $4.90 \times 10^{3}$ & $2+$ & $4+4+4+$ & $3+$ \\
$10^{-3}$ & $4.60 \times 10^{2}$ & $1+$ & $1+2+$ & $2+$ & $1+$ \\
$10^{-4}$ & $4.90 \times 10^{1}$ & - & - & - & \pm & -
\end{tabular}

*Intensity of agglutinations graded as follows: $1+$, very slight, fine granular; $2+$, moderate agglutination with uniform small clumps; $3+$, heavy agglutination with uniform large clumps; $4+$, heavy agglutination with widely scattered large clumps and clearing of suspending fluid.

culture will be performed. If no culture will be done, the direct antigen detection test may be used to supplement the physician's physical findings. The test could provide a rapid means of improving the diagnosis of GAS pharyngitis and increase the chance of appropriate therapy.

The results of this study showed that the five DLAKs tested performed equally well with regard to sensitivity and specificity. The choice of which kit to use could be based on cost and ease of operation.

1. Facklam RR: Streptococci and aerococci, in Lennette EH (ed in chief): Manual of Clinical Microbiology, ed 3. Washington, D.C., American Society for Microbiology, 1980 , p 88 .

2. Facklam RR: Isolation and Identification of Streptococci. Atlanta, Centers for Disease Control, 1980, p 54.

3. Kurzynski TA, Van Holten CM: Evaluation of techniques for isolation of group A streptococci from throat cultures. J Clin Microbiol 1981;13:891-894.

4. Lancefield RC: A serologic differentiation of human and other groups of hemolytic streptococci. J Exp Med 1933;57:571-95.

5. Castle, D, Kessock-Philip S, Easmon CSF: Evaluation of an improved Streptex kit for the grouping of beta-haemolytic streptococci by agglutination. J Clin Pathol 1982;35:719-722.

6. Baker CJ, Rench MA: Commercial latex agglutination for detection of group B streptococcal antigen in body fluids. J Pediatr 1983;102:393395.

7. Edwards EA, Phillips IA, Suiter WC: Diagnosis of group A streptococcal infections directly from throat secretions. J Clin Microbiol 1982;15:481. 483.

8. Petts DN: Early detection of streptococci in swabs by latex agglutination before cultures. J Clin Microbiol 1984;19:432-433.

9. Sippel JE et al: Use of the Directigen latex agglutination test for detection of Haemophilus influenzae, Streptococcus pneumoniae, and Neisseria meningitidis antigen in cerebrospinal fluid from meningitis patients. J Clin Microbiol 1984;20:884-886.

10. Campos JM, Charilaou CC: Evaluation of Detect-A-Strep and the Culturette ten-minute strep ID kits for detection of group A streptococcal antigen in oropharyngeal swabs from children. J Clin Microbiol 1985;22:145-148.

11. Miceika BG, Vitous AS, Thompson KD: Detection of group A streptococcal antigen directly from throat swabs with a ten-minute latex agglutination test. J Clin Microbiol 1985;21:467-469.

12. Kamm W, Bille J: Evaluation of a rapid latex agglutination test (Directigen) for the direct detection of group A streptococci from throat swabs. Infection 1985;13 (Jul-Aug):179-181.

13. Venezia RA, Ryan A, Alward S: Evaluation of a rapid method for the detection of streptococcal group A antigen directly from throat swabs. J Clin Microbiol 1985;21:395-398.

14. Berkowitz CD, Anthony BF, Kaplan EL: Cooperative study of latex 
agglutination to identify group A streptococcal antigen on throat swabs in patients with acute pharyngitis. $J$ Pediatr 1985; 107:89-92.

15. Gerber MA, Spadoccini LJ, Wright LL et al: Latex agglutination tests for rapid identification of group A streptococci directly from throat swabs. J Pediatr 1984;105:702-705.

16. McCusker JJ, McCoy EL, Young CL et al: Comparison of Directigen group A strep test with a traditional culture technique for detection of group A beta-hemolytic streptococci. J Clin Microbiol 1984;20:824-825.

17. Miller JM Phillips HL, Graves RK et al: Evaluation of the Directigen group A strep test kit. J Clin Microbiol 1984;20:846-848.

18. Slifkin M, Gil GM: Evaluation of the Culturette brand ten-minute group A strep ID technique. J Clin Microbiol 1984;20:12-14.

19. Radetsky M, Wheeler RC, Roe $\mathrm{MH}$ et al: Comparative evaluation of kits for rapid diagnosis of group A streptococcal disease. Pediatr Infect Dis 1985;4:274-281.

20. Aldridge KE, Kogos C, Sanders CV et al: Comparison of rapid identification assays for Staphylococcus aureus. J Clin Microbiol 1984;19:703. 704 .

21. Baker JS, Bormann MA, Boudreau DH: Evaluation of various rapid agglutination methods for the identification of Staphylococcus aureus. $J$ Clin Microbiol 1985;21:726-729.

22. Field, PR, Gong CM: Diagnosis of postnatally acquired rubella by use of three enzyme-linked immunosorbent assays for specific immunoglobulins $\mathrm{G}$ and $\mathrm{M}$ and single radial hemolysis for specific immunoglobulin G. J Clin Microbiol 1984;20:951-958.

23. Kleeman KT, Kiefer DJ, Halbert SP: Rubella antibodies detected by several commercial immunoassays in hemagglutination inhibitionnegative sera. J Clin Microbiol 1983;18:1131-1137.

24. Tilton RC, Dias F, Ryan RW: Comparative evaluation of three commercial products and counterimmunoelectrophoresis for the detection of antigens in cerebrospinal fluid. J Clin Microbiol 1984;20:231-234.

25. Griner PF, Mayewski RJ, Mushlin AI et al: Selection and interpretation of diagnostic tests and procedures: Principles and applications. Ann Intern Med 1981:94(Pt 1):557-600.
26. Forrer CB, Ellner PD: Distribution of hemolytic streptococci in respiratory specimens. $J$ Clin Microbiol 1979;10:69-71.

27. Kelly MT et al: Outpatient evaluation of a rapid, direct test for detection of group A streptococci in throat swabs. Abstr Ann Mtg Am Soc Microbiol, 1985; p 127.

28. Benjamin JT, Perriello VA Jr: Pharyngitis due to group C hemolytic streptocci in children. $J$ Pediatr 1976;89:254-256.

29. Rolston KVI: Group G streptococcal infections, editorial. Arch Intern Med 1986;146:857-858.

30. Stamm AM, Cobbs CG: Group C streptococcal pneumonia: Report of a fatal case and review of the literature. Rev Infect Dis 1980;2:889. 898.

31. McCue JD: Group G streptococcal pharyngitis: Analysis of an outbreak at a college. JAMA 1982;248:1333-1336.

32. Krober MS, Bass JW, Michels GN: Streptococcal pharyngitis: Placebocontrolled double-blind evaluation of clinical response to penicillin therapy. JAMA $1985 ; 253: 1271-1274$

33. Kellogg JA, Manzella JP: Detection of group A streptococci in laboratory or physician's office: Culture vs. antibody methods. JAMA 1986;255:2638-2642.

34. Carlson, JR, Huston AC, Watson TE et al: Development and evaluation of Detect-A-Strep ${ }^{\pi}$ : A new rapid latex agglutination kit for the direct detection of group A streptococcal antigen in throat. Washington, D.C., Oct 84, p 92.

Dr Burdick is associate professor of microbiology and Dr Sottile is adjunct associate professor of pathology at the Chicago College of Osteopathic Medicine, Chicago, Il.

Dr Burdick, Department of Microbiology, Chicago College of Osteopathic Medicine, 5200 S Ellis Ave, Chicago, Il 60615. 
EW FROM MILES
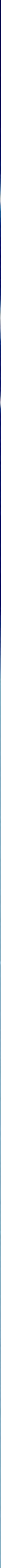


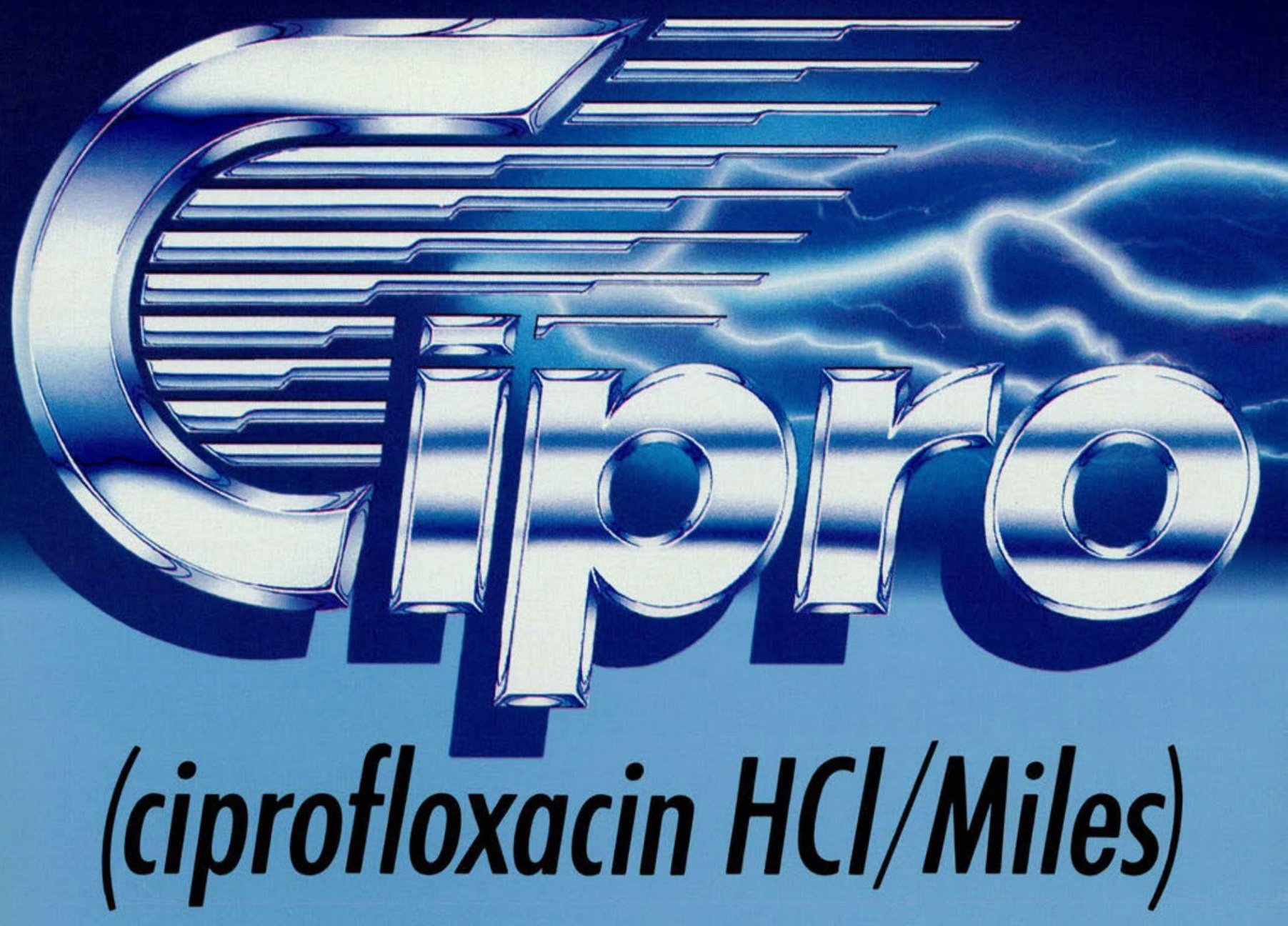

\section{THE MOST POTENT QUINOLONE'-3*}

*In vitro activity does not necessarily imply a correlation with in vivo results. 


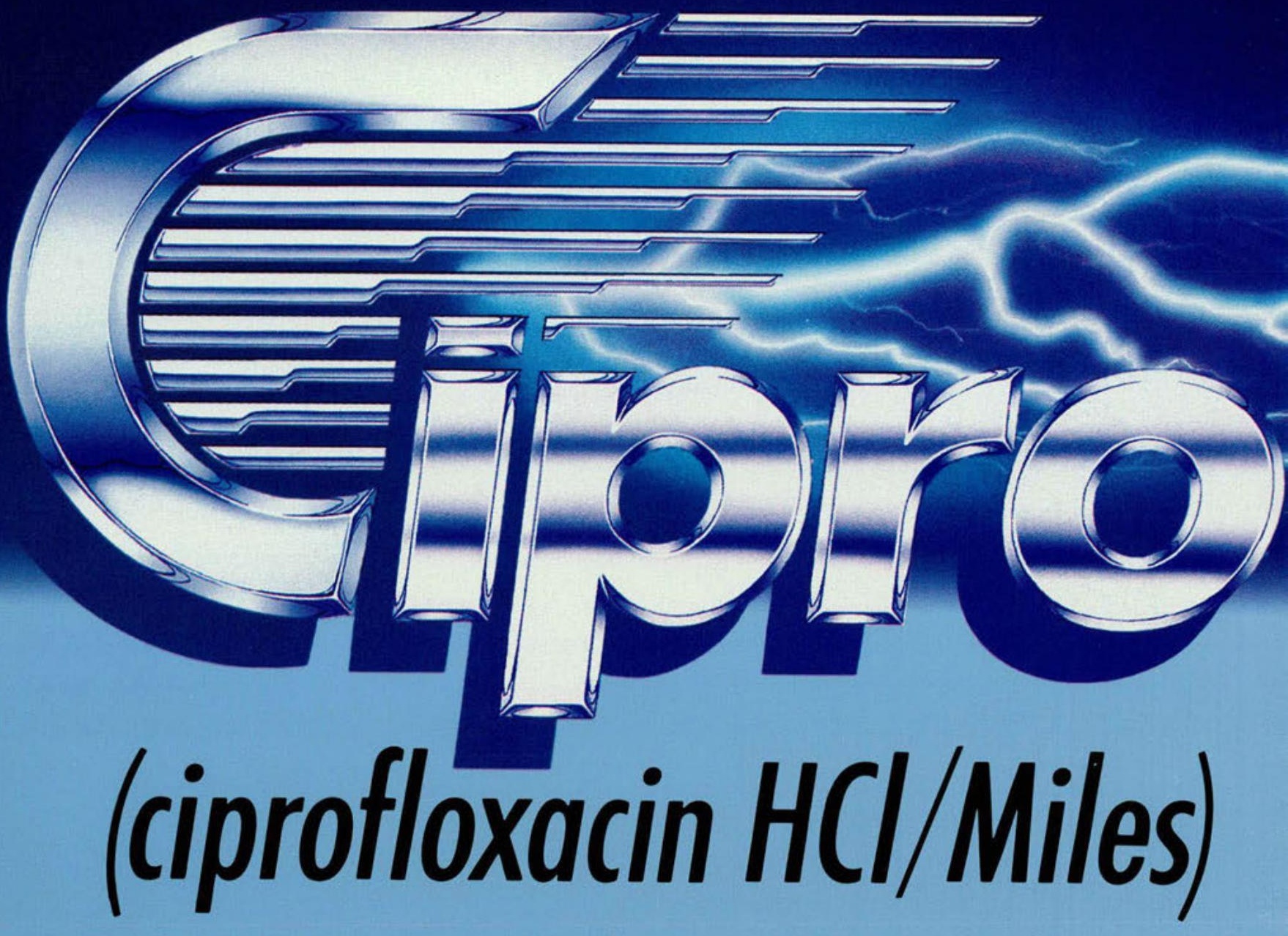

CONVENIENT

B.I.D. DOSAGE

$250 \mathrm{mg}, 500 \mathrm{mg}$, and $750 \mathrm{mg}$ tablets 

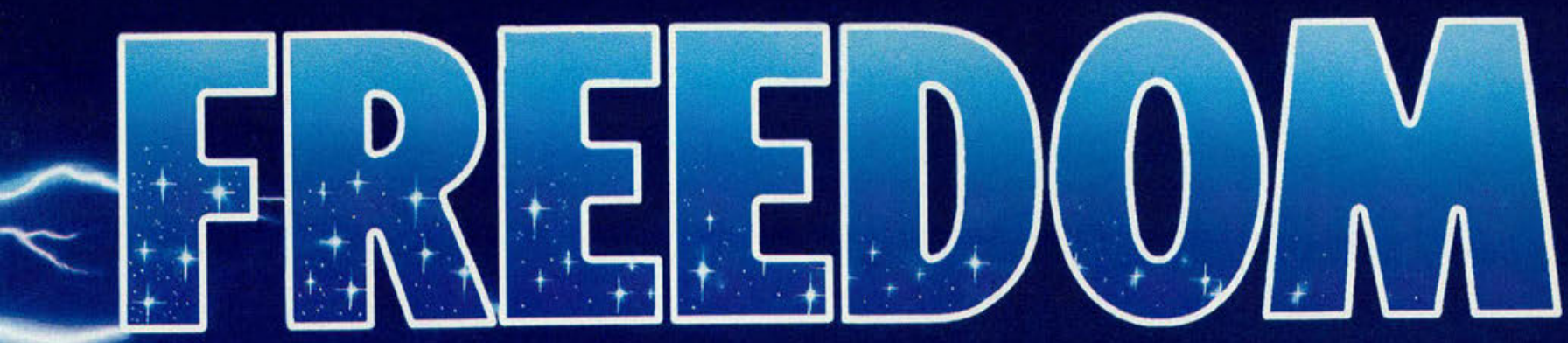

\section{THE POWER OF PAREIIIERALS. THIE FREADOM OF ORAL DOSAGE.}

\section{- Beyond the limitations of parenteral antimicrobials and traditional oral therapy}

- For outpatient treatment of many infections that previously required hospitalization

\section{Well tolerated-low incidence of side effects}

CIPRO ${ }_{\text {SHOULD NOT BE USED IN CHILDREN OR PREGNANT WOMEN. }}$

Notes: Concomitant administration of ciprofloxacin with theophylline moy prolong the serum elimination half-life of theophylline, elevote serum theophylline levels, and may increase the risk of adverse reactions (see precoutions).

Antocids containing magnesium hydroxide or aluminum hydroxide may interfere with the obsorption of ciprofloxacin, resulting in serum and urine levels lower than desired: concurrent odministrotion of these ogents with ciprofloxocin should be ovoided.

A history of hypersensitivity to ciprofloxocin is o controindication to its use. A history of hypersensitivity to other quinolones may also controindicate the use of ciprofloxacin.

For further information, contact the Miles Information Service: 1-800-642-4776.

In VA. call collect: 703-620-2799.
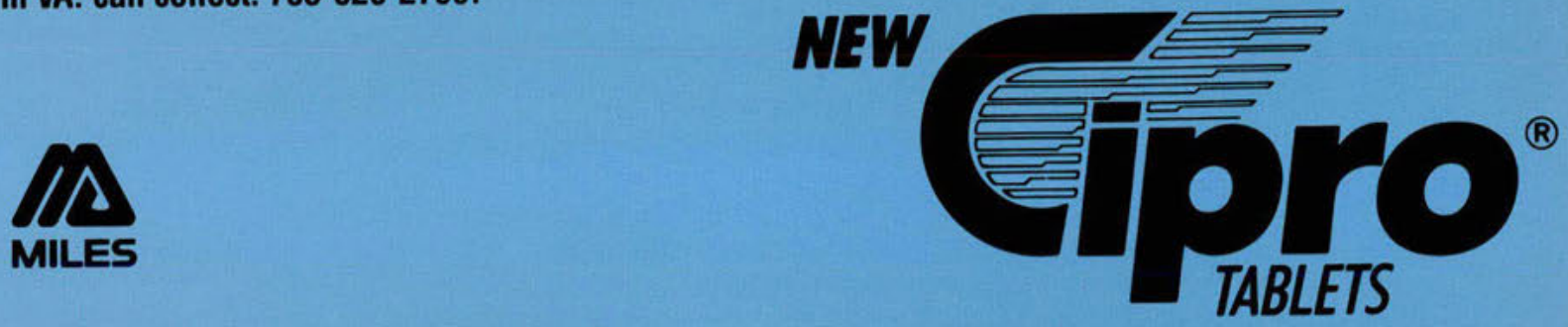
NEW

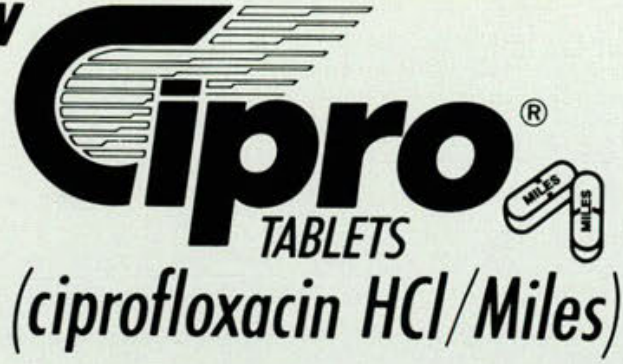

CONSULT PACKAGE INSERT FOR FULL PRESCRIBING INFORMATION

Cipro is indicated for the treatment of the following infections caused by susceptible strains of the indicated microorganisms: lower respiratory, skin and skin structure, bone and joint, and urinary tract; and infectious diarthea (SEE FULL PRESCRIBING INFORMATION FOR SPECIFIC ORGANISMS) CONTRAINDICATIONS

A history of hypersensitivity to ciprofloxacin is a contraindication to its use. A history of hypersensitivity to other quinolones may also contraindicate the use of ciprofiloxacin.

CIPROFLOXACIN SHOULD NOT BE USED IN CHILDREN OR PREGNANT WOMEN. The oral administration of ciprofloxacin caused lameness in immature dogs. Histopathological examination of the weight-bearing joints of these dogs revealed permanent lesions of the cartilage. Related drugs such as nalidixic acid, cinoxacin. and norfloxacin also produced erosions of cartilage of weight-bearing joints and other signs of arthropathy in immature animals of various species ISEE ANIMAL PHARMACOLOGY SECTION IN FULL PRESCRIBING INFORMATION).

General:

\section{PRECAUTIONS}

As with other quinolones, ciprofloxacin may cause central nervous system (CNS) stimulation, which may lead to tremor, restlessness, lightheadedness, confusion, and very rarely to hallucinations or convulsive seizures

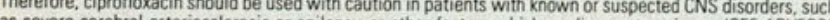
as severe cerebral arteriosclerosis or epilepsy, or other factors which predispose to seizures ISEE ADVERSE

Crystals of ciprofloxacin have been observed rarely in the urine of human subjects but more frequently in the urine of laboratory animals. Crystalluria related to ciprofloxacin has been reported only rarely in man, because human unine is usualy acidic. Patients recerving ciprofolloxacin should be well hydrated, and alkalinity of the urine should be avoided. The recommended daily dose should not be exceeded. Alteration of the diosage regimen is necessary for patients with impairment of renal function (SEE DOSAGE AND ADMINISTRATION)

Drug Interactions:

Concurrent administration of ciprofloxacin with theophylline may lead to elevated plasma concentrations of theophylline and prolongation of its elimination half-life. This may result in increased risk of theophylline-related adverse reactions. If concomitant use cannot be avoided, plasma levels of theophylline should be monitored and dosage adjustments made as appropriate.

Antacids containing magnesium hydroxide or aluminum hydroxide may interfere with the absorption of ciprofloxacin, resulting in serum and urine levels lower than desired; concurrent administration of these agents with ciprofloxacin should be avoided

Probenecid interferes with the renal tubular secretion of ciprofloxacin and produces an increase in the level of ciprofloxacin in the serum. This should be considered if patients are receiving both drugs concomitantly
As with other broad-spectrum antibiotics, As with other broad-spectrum antibiotics, prolonged use of ciprofloxacin may result in overgrowth of nonsus. ceptible organisms. Repeated evaluation of the patient's condition and microbial susceptibility testing is essential. If superinfection occurs during therapy, appropriate measures should be taken

Patients should be advised that ciprofloxacin may be taken with or without meals. The preferred time of dosing is two hours after a meal. Patients should also be advised to drink fluids liberally and not take antacids containing magnesium or aluminum concomitantly or within two hours after dosing. Ciprofloxacin may cause dizziness or lightheadedness, therefore patients should know how they react to this drug before they operate an automobile or machinery or engage in activities requiring mental alertness or coordination.

Carcinogenesis, Mutagenesis, Impairment of Fertility

Eight in vitro mutagenicity tests have been conducted with ciprofloxacin and the test results are listed below. Salmonella/Microsome Test (Negative)

E. coli DNA Repair Assay (Negative)

Mouse Lymphoma Cell Forward Mutation Assay (Positive)

Chinese Hamster $\mathrm{V}_{79}$ Cell HGPRT Test (Negative)

Syrian Hamster Embryo Cell Transformation Assay (Negative)

Saccharomyces cerevisiae Point Mutation Assay (Negative)

Saccharomyces cerevisiae Mitotic Crossover and Gene Conversion Assay (Negative)

Rat Hepatocyte DNA Repair Assay (Positive)

Thus, two of the eight tests were positive, but the following three in vivo test systems gave negative results Rat Hepatocyte DNA Repair Assay

Micronucleus Test (Mice)

Dominant Lethal Test (Mice)

Long-term carcinogenicity studies in animals have not yet been completed.

Pregnancy-Pregnancy Category $C$ :

heproduction studies have been performed in rats and mice at doses up to six times the usual daily human dose and have revealed no evidence of inpaired ferttility orharm to the fetus due to ciprofloxacin. In rabbits, as with most antimicrobial agents. ciproflloxacin $(30$ and $100 \mathrm{mg} / \mathrm{kg}$ orally) produced gastrointestinal disturbances re sulting in maternal weight loss and an increased incidence of abortion. No teratogenicity was observed at either dose. After intravenous administration, at doses up to $20 \mathrm{mg} / \mathrm{kg}$. no maternal toxicity was produced, and no em. bryotoxicity or teratogenicity was observed. There are, however, no adequate and well-controlled studies in pregnant women. SINCECIPROFLOXACIN, UKE OTHER DRUGS IN ITS CLASS, CAUSES ARTHROPATHY IN IMMA. IURE ANIMALS, IT SHOULD NOT BE USED IN PREGNANT WOMEN (SEE WARNINGS)

Nursing Mothers

It is not known whether ciprofloxacin is excreted in human milk; however, it is known that ciprofloxacin is excreted in the mik of actating gats and that other drugs of this class are excreted in human milk. Because of this and because of the potential for serious adverse reactions from ciprofloxacin in nursing infants, a decision should be made to discontinue nursing or to discontinue the drug, taking into account the importance of the drug to the mother:

Pediatric Use:

Ciprofloxacin should not be used in children because it causes arthropathy in immature animals ISEE WARNINGS).

\section{ADVERSE REACTIONS}

Ciprofloxacin is generally well-tolerated. During clinical investigation, 2,799 patients received 2.868 courses of the drug. Adverse events that were considered likely to be drug related occurred in $7.3 \%$ of courses, possibly re. lated in $9.2 \%$, and remotely related in $3.0 \%$. Ciprofloxacin was discontinued because of an adverse event in $3.5 \%$ of courses, primarily involving the gastrointestinal system $(1.5 \%)$, skin $(0.6 \%)$, and central nervous system $(0.4 \%)$

The most frequently reported events, drug related or not, were nausea $(5.2 \%)$, diarrhea $(2.3 \%)$, vomiting (2.0\%), abdominal pain/discomfort (1.7\%), headache $(1.2 \%)$, restlessness $(1.1 \%)$, and rash (1.1\%)

Additional events that occurred in less than $1 \%$ of ciprofloxacin courses are listed below. Those typical of quinolones are italicized.

\section{CONVENIENT B.I.D. DOSAGE}

\section{Recommended dosage schedule}

Severity of

Infection

Dosage

\begin{tabular}{lcc} 
Infection Site & Infection & Dosage \\
\hline Respiratory Tract & Mild/Moderate & $500 \mathrm{mg}$ B.I.D. \\
\cline { 2 - 3 } Bone and Joint \\
Skin/Skin Structure & Severe/Complicated & $750 \mathrm{mg}$ B.I.D. \\
\hline Urinary Tract & Mild/Moderate & $250 \mathrm{mg}$ B.I.D. \\
\cline { 2 - 3 } & Severe/Complicated & $500 \mathrm{mg}$ B.I.D. \\
\hline Infectious Diarrhea & Mild/Moderate/Severe & $500 \mathrm{mg}$ B.I.D. \\
\hline
\end{tabular}

GASTROINTESTINAL: (See above), painful oral mucosa, oral candidiasis, dysphagia, intestinal perforation, gastrointestinal bleeding.

CENTRAL NERVOUS SYSTEM: (See above), dizziness, lightheadedness, insomnia, nightmares, hallucinations, manic reaction, irritability, tremor, ataxia, convulsive seizures, lethargy, drowsiness, weakness, malaise, anorexia, phobia, depersonalization, depression, paresthesia.

(See above), pruritus, urticaria, photosensitivity, flushing fever, chills, angiodema, edema of the face, neck, lips, conjunctivae or hands, cutaneous candidiasis, hyperpigmentation, erythema nodosum.

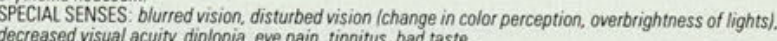
decreased visual acuity, diphopia, eve pain vinion lch, bad taste.

RENAL UUROGENITAL. , polyuria, urinary retention, urethra bleeding, vaginitis, acidosis

s, atrial flutter, ventricular ectopy, syncope, hypertension, angina pectoris, myocardial infarction, cardiopulmonary arrest, cerebral thrombosis.

RESPIRATORY: epistaxis, laryngeal or pulmonary edema, hiccough, hemoptysis, dyspnea, bronchospasm. pulmonary embolism.

Most of these events were described as only mild or moderate in severity, abated soon after the drug was dis.

Insed, and required no treatment.

In several instances, nausea, vomiting, tremor, restlessness, agitation, or palpitations were judged by investigators to be related to elevated plasma levels of theophylline possibly as a result of a drug interaction with ci-

Adverse Laboratory Changes: Changes in laboratory parameters listed as adverse events without regard to drug

Hepatic-Elevations of: ALT (SGPT) (1.9\%), AST (SGOT) (1.7\%), Alkaline Phosphatase $(0.8 \%)$, LOH $(0.4 \%)$,

Hematologic-eosinophilia $(0.6 \%)$, leukopenia $(0.4 \%)$, decreased blood platelets $(0.1 \%)$, elevated blood platelets $(0.1 \%)$, pancytopenia $(0.1 \%$.

Renal-Elevations of: Serum creatinine (1.1\%). BUN $10.9 \%$

BEEN REPORTED.

Other changes occurring in less than $0.1 \%$ of courses were: Elevation of serum gammaglutamyl transferase, elevation of serum amylase, reduction in blood glucose, elevated uric acid, decrease in hemoglobin, anemia, bleeding diathesis, increase in blood monocytes, and leukocytosis.
OVERDOSAGE

Information on overdosage in humans is not available. In the event of acute overdosage, the stomach should be emptied by inducing vomiting or by gastric lavage. The patient should be carefully observed and given supportive reatment. Adequate hydration must be maintained. In the event of serious toxic reactions from overdosage, hemodialysis or peritoneal dialysis may aid in the removal of ciprofloxacin from the body, particularly if renal funcion is compromised

\section{DOSAGE AND ADMINISTRATION}

The usual adult dosage for patients with urinary tract infections is $250 \mathrm{mg}$ every 12 hours. For patients with com licated infections caused by organisms not highly susceptible, $500 \mathrm{mg}$ may be administered every 12 hours. Respiratory tractinfections, skin and skin structure infections, and bone and joint infections may be treated with $500 \mathrm{mg}$ every 12 hours. For more severe or complicated infections, a dosage of $750 \mathrm{mg}$ may be given every 12 hours.

The recommended dosage for infectious diarrhea is $500 \mathrm{mg}$ every 12 hours.

In patients with renal impairment, some modification of dosage is recommended (SEE DOSAGE AND ADMIN. ISTRATION SECTION IN FULL PRESCRIBING INFORMATION. HOW SUPPLIED

Cipro* (ciprofloxacin HCI/Miles) is available as tablets of $250 \mathrm{mg}$. $500 \mathrm{mg}$. and $750 \mathrm{mg}$ in bottles of 50 , and in Unit-Dose packages of 100 (SEE FULL PRESCRIBING INFORMATION FOR COMPLETE DESCRIPTION).

References: 1. Barry. A.L., Jones, R.N., Thornsberry, C., et al.: Antibacterial activities of ciprofloxacin, norfloxacin, oxolinic acid, cinoxacin, and nalidixic acid. Antimicrob. Agents Chemother. 25:633-637, 1984. 2. Guimaraes, M.A., and Noone. P.: The comparative in-vitro activity of norfloxacin, ciprofloxacin, enoxacin and nalidixic acid against 423 strains of gram-negative rods and staphylococci isolated from infected hospitalised patients. J. Antimicrob. Chemother. 17:63-67, 1986. 3. Van Caekenberghe, D.L., and Pattyn, S. R.: In vitro activity of ciprofloxacin compared with those of other new fluorinated piperazinyl-substituted quinoline derivatives. Antimicrob. Agents Chemother. 25:518-521, 1984 .

For further information, contact the Miles Information Service: 1-800-642-4776. In VA. call collect: 703-620-2799.

\section{COMMITTED TO THERAPEUTIC EFFICIENCY}

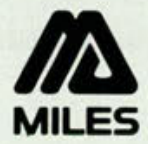

Miles Inc Pharmaceutical Division 400 Morgan Lane West Haven, CT 06516
C09047 MLD-697

Printed in U.S.A 